\title{
Canadian Public Health Laboratory Network Statement on Point-of-Care Serology Testing in COVID-19
}

\author{
Respiratory Virus Infections Working Group ${ }^{1}$
}

\author{
Suggested citation: Respiratory Virus Infections Working Group. Canadian Public Health Laboratory Network \\ Statement on Point-of-Care Serology Testing in COVID-19. Can Commun Dis Rep 2020;46(5):119-20. \\ https://doi.org/10.14745/ccdr.v46i05a03
}

Keywords: COVID-19, point-of-care, serology testing, Canada, antibodies to SARS-CoV-2

\section{Introduction}

Point-of-care (POC) serology testing for severe acute respiratory syndrome coronavirus 2 (SARS-CoV-2), the virus that causes COVID-19, detect the human antibody response to infection rather than the virus itself. Most POC serology tests are qualitative immunochromatographic (lateral-flow) based assays that detect immunoglobulins $\mathrm{M}$ and/or $\mathrm{G}$ in blood from a finger prick and can provide results in less than 30 minutes. While there is widespread interest in adopting POC serology tests for SARS-CoV-2, there are currently significant limitations to this testing modality, including the lack of understanding of the immunological response in COVID-19, limited clinical validation data and variability in performance among different POC tests.

\section{Current position for use of point-of-care serology testing for acute diagnostics}

The POC serology tests for SARS-CoV-2 have not currently been validated for use as a diagnostic tool for acute infection and none is approved by Health Canada to date. In general, these antibody tests often do not become positive until a week or more after symptoms have started and, therefore, are not suitable for diagnosis of acute SARS-CoV-2 infection at this time. We recommend that nucleic acid detection (e.g. real-time polymerase chain reaction, $P C R$ ) remains the first-line test for the diagnosis of acute SARS-CoV-2 infection, as advised by the World Health Organization (1).

\section{Key points relating to point-of-care serology testing}

- It can take 7-12 days after symptom onset for antibodies to SARS-CoV-2 to develop; therefore, the use of POC serology tests in the early phase of infection can result in falsenegative results at a time when patients are most infectious (i.e. a negative result does not rule out infection) Since POC serology tests do not detect virus, a positive or negative
This work is licensed under a Creative Commons Attribution 4.0 International License.

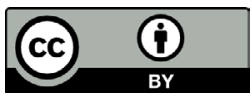

Affiliation

${ }^{1}$ Canadian Public Health Laboratory Network, Winnipeg, MB

${ }^{\star}$ Correspondence:

dionne.marcino@canada.ca result does not determine whether a person is infectious

- Positive results may reflect either a past or present infection with SARS-CoV-2

- $\quad$ False-positive results may occur if these kits cross react with antibodies from recent or past exposure to other coronaviruses, including human seasonal coronaviruses (HKU1, NL63, OC43, 229E), severe acute respiratory syndrome coronavirus 1 (SARS-CoV-1) or Middle East respiratory syndrome coronavirus (MERS-CoV). Other infections, as well as non-infectious conditions (e.g. rheumatoid factor-positive diseases) may also cause falsepositive results. All kits considered for use need to be thoroughly evaluated for such cross reactivity before being used clinically

- $\quad$ False-negative results may occur in elderly and immunocompromised patients

\section{Where point-of-care serology testing could be used}

At present, the use of serology in the diagnosis of acute SARS-CoV-2 infection and patient management is likely to be of limited utility. However, once the dynamics of the serological response in COVID-19 are better understood, serology will play an important role in the public health response. A key aspect of the use of serology testing is understanding whether antibody production correlates with protective immunity and what the duration of that protection is. The ease of use and quick turnaround time of POC assays make it an ideal testing modality in 1) remote areas with limited access to centralized laboratorybased testing and/or local laboratory infrastructure and 
2) situations that would benefit from immediate triaging.

Examples of the latter include the following:

- seroepidemiology-used to better understand the proportion of undiagnosed in the population over time and to provide more accurate data of attack and mortality rates

- $\quad$ informing targeted diagnostic testing strategies (using PCR testing), where priority would be given to populations/areas with no evidence of immunity

- detecting seroconversion and assessing immunity in healthcare workers and other essential/frontline workers

- as an adjunct to PCR for diagnostic testing in patients who are PCR-negative and in the late course of their illness to implement control measures and to effectively manage patients

- $\quad$ testing high-risk populations exposed to SARS-CoV-2 to assess their risk of developing infection

- detecting seroconversion as a surrogate for effectiveness of control measures

- determining, once a vaccine is available, who should be prioritized for earlier vaccination

- supporting clinical trials that are assessing novel therapies, such as the use of neutralizing antibodies

\section{Important considerations for implementing point-of-care serology testing}

- A well validated test, which has been evaluated against a gold standard (viral neutralization assays or another laboratory-based serological assay), is required. Performance characteristics (sensitivity, specificity, positive and negative predictive values, cross-reaction to other coronaviruses) should be established using sera from 1) patients infected with SARS-CoV-2, 2) other respiratory viruses, including seasonal coronaviruses, and 3) healthy controls

- Adequate training of healthcare workers to administer the test and interpret the result will be required

- $\quad$ Risk of infection with SARS-CoV-2 and bloodborne infections for the operator must be assessed

- Provisions must be in place to ensure the capture of 1) testing data for individual patient records and surveillance purposes and 2) requirement for participation in external quality assessment to maintain high-quality testing

\section{Conclusion}

Based on currently available information, the Canadian Public Health Laboratory Network recommends that SARS-CoV-2 POC serological assays not be used for clinical testing in any capacity at this time. As more information becomes available on test performance, and assays are validated against gold standard serological methods, clinical application of POC assays will be reevaluated. Molecular testing, such as real-time PCR, remains the primary test method for laboratory confirmation of acute SARS-CoV-2 infection and diagnosis of COVID-19.

\section{Authors' statement}

The Respiratory Virus Infection Working Group of the Canadian Public Health Laboratory Network (CPHLN) is dedicated to providing leadership and guidance on topics related to respiratory viral pathogens, including laboratory response to emerging respiratory viruses. The Respiratory Virus Infection Working Group is comprised of leaders from public health laboratories across Canada.

\section{Acknowledgements}

The Respiratory Virus Infection Working Group would like to thank members of the Canadian Public Health Laboratory Network (CPHLN) Secretariat, S Radons Arneson and D Marcino, for coordination of document synthesis. We would also like to thank the Laboratory Directors Council of the CPHLN for review of the document.

\section{Reference}

1. World Health Organization. Advice on the use of point-of-care immunodiagnostic tests for COVID-19. WHO; 2020. https:// www.who.int/news-room/commentaries/detail/advice-on-the-us e-of-point-of-care-immunodiagnostic-tests-for-covid-19 